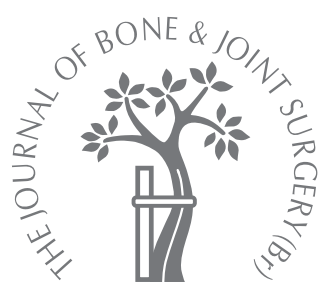

D. K. Bae,

S. J. Song,

K. H. Yoon

From Department of

Orthopaedic Surgery,

School of Medicine,

Kyung Hee

University, Seoul,

Korea

D. K. Bae, MD, $\mathrm{PhD}$, Professor

I S. J. Song, $\mathrm{MD}, \mathrm{PhD}$

Associate Professor

K. H. Yoon, MD, PhD

Associate Professor

Department of Orthopaedic

Surgery, School of Medicine

Kyung Hee University, 1 Hoeki-

dong, Dongdaemun-ku, Seoul

130-702, Korea.

Correspondence should be sent

to Dr S. J. Song; e-mail:

songsjun@khmc.or.kr

(C)2009 British Editorial Society

of Bone and Joint Surgery

doi:10.1302/0301-620X.91B9.

$22058 \$ 2.00$

$J$ Bone Joint Surg [Br]

2009;90-B:1164-71.

Received 13 November 2008;

Accepted after revision 8 April

2009

\title{
Closed-wedge high tibial osteotomy using computer-assisted surgery compared to the conventional technique
}

\begin{abstract}
We assessed the reliability, accuracy and variability of closed-wedge high tibial osteotomy (HTO) using computer-assisted surgery compared to the conventional technique. A total of 50 closed-wedge HTO procedures were performed using the navigation system, and compared with $\mathbf{5 0}$ HTOs that had been performed with the conventional technique. In the navigation group, the mean mechanical axis prior to osteotomy was varus $8.2^{\circ}$, and the mean mechanical axis following fixation was valgus $3.6^{\circ}$. On the radiographs the mean preoperative mechanical axis was varus $7.3^{\circ}$, and the mean post-operative mechanical axis was valgus $2.1^{\circ}$. There was a positive correlation between the measured data taken under navigation and by radiographs $(r>0.3, p<0.05)$. The mean correction angle was significantly more accurate in the navigation group $(p<0.002)$. The variability of the correction was significantly lower in the navigation group $\left(2.3^{\circ}\right.$ vs $\left.3.7^{\circ}, p=0,012\right)$.

We conclude that navigation provides reliable real-time intra-operative information, may increase accuracy, and improves the precision of a closed-wedge HTO.
\end{abstract}

The aim of a high tibial osteotomy (HTO) is to change the mechanical axis of the lower limb and thereby transfer the body weight across healthy articular cartilage. ${ }^{1,2}$ There are two basic techniques: the medial open-wedge and the lateral closed-wedge osteotomy. ${ }^{3}$ Advantages of the lateral closed-wedge osteotomy include the presence of broad metaphyseal opposing surfaces, offering quicker healing ${ }^{1}$ and negating the risk of loss of correction, fixation failure, delayed union and nonunion and satisfactory long-term results have been reported. ${ }^{4-8}$ However, there are potential risks of neurovascular injury and of creating additional difficulty when converting a knee with a previous osteotomy to a total knee replacement (TKR). ${ }^{9,10}$

Several long-term studies indicate that accurate correction has been the leading predictor for success. ${ }^{4-6,10}$ Conventional methods for determining the correction angle and wedge size include pre-operative radiological measurement and planning, intra-operative measurement with the help of a cable, grids with lead-impregnated reference lines, and a system that uses a jig. ${ }^{4,11}$ However, it is difficult to obtain the ideal correction with conventional techniques because of the unreliability of preoperative planning. Occasionally, the conventional full-length leg radiograph is also inaccurate because of rotation of the lower extremity, and image parallax may also introduce a measurement error. ${ }^{12-14}$ Moreover, determination of the wedge size depends on more than a static measurement of the deformity: it also needs to be based on dynamic angle analysis and the position of the osteotomy plane, as well as the size of the patient. Besides the determination of wedge size, there are other problems with the conventional technique, such as alteration of the tibial posterior slope angle ${ }^{15}$ and penetration of the medial cortical bone. ${ }^{16-18}$ Navigation offers the possibility of increasing the accuracy, precision and repeatability of many orthopaedic practices. There have been a small number of studies reporting open-wedge HTO using the navigation system, ${ }^{19,20}$ but there are very few clinical reports confirming the results of the closed-wedge HTO using this system.

The aim of our study was to compare the measurement data obtained using navigation with radiological measurements in a closedwedge navigated HTO, thereby assessing its reliability. Our secondary aim was to analyse the radiological results of a closed-wedge HTO using computer-assisted surgery compared with the conventional method, and to assess levels of accuracy and variability.

\section{Patients and Methods}

Between July 2005 and July 2006, 50 closedwedge HTO procedures were performed and 
Table I. Demographic data of the navigated and the conventional groups

\begin{tabular}{llll}
\hline & Navigated group & Conventional group & p-value \\
\hline Patients & 48 & 34 & \\
Knees & 50 & 50 & \\
Age in yrs (range) & $59.4(48$ to 71$)$ & $60.7(45$ to 76$)$ & 0.599 \\
Body mass index (SD) & $24.9(2.8)$ & $25.4(3.6)$ & 0.419 \\
Female:male & $44: 4$ & $31: 3$ & \\
Right:left & $30: 20$ & $26: 24$ & \\
Osteoarthritis:genu varum & $48: 2$ & $43: 7$ & \\
\hline
\end{tabular}
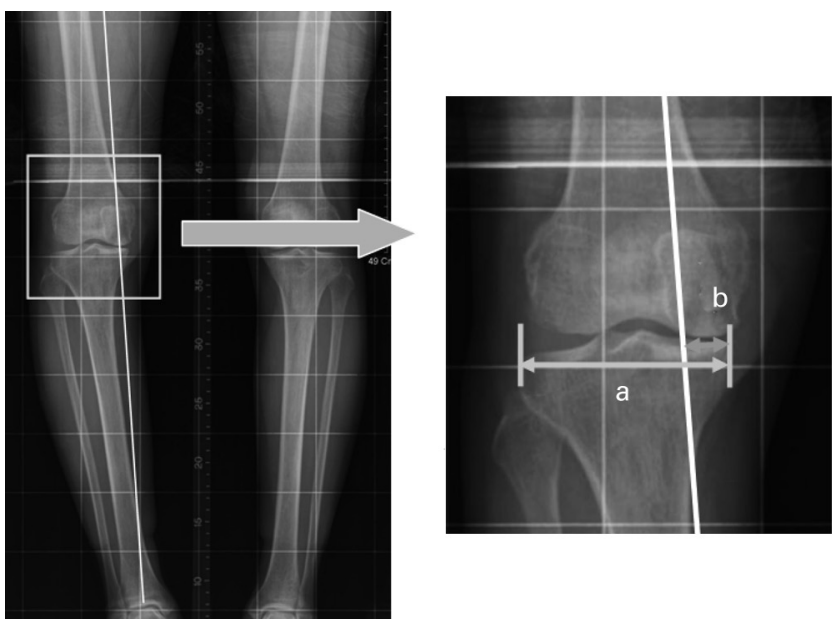
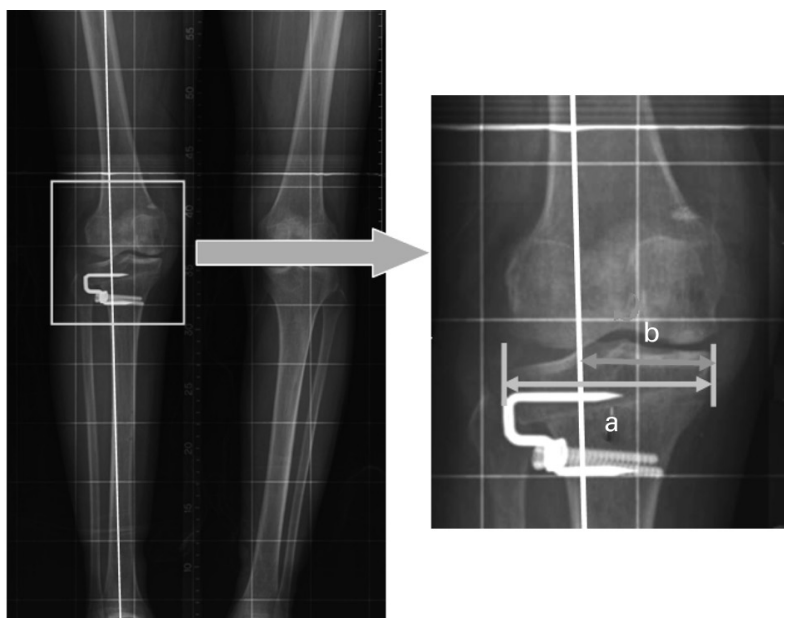

Fig. 1

Radiographs showing a) pre-operative medial deviation of the mechanical axis and b) post-operative lateral deviation of the mechanical axis. The mechanical axis percentage shown on the orthoroentgenogram was evaluated by percentile deviation (b/a) $\times 100]$. (a) is the width of tibia plateau and (b) is the distance from the medial border of the medial tibial condyle to the point at which the mechanical axis intersects the knee joint line.

analysed using the CT-free navigation system (Vector Vision version 1.1, BrainLAB, Heimstetten, Germany), for medial compartment osteoarthritis of the knee and genu varum. A total of 50 closed-wedge HTOs using the conventional technique performed between January 1994 and July 2006, were retrospectively reviewed as a control group. All the cases had good-quality pre-operative and follow-up radiographs. A miniplate staple (U\&I, Uijungbu-si, Korea) ${ }^{4,5}$ was used to fix the osteotomy in both the navigated and the conventional procedures. Informed consent was obtained from all patients before the review. The mean age of the patients was 59.4 years (48 to 71$)$ for the navigation group and 60.7 years (45 to 76) for the conventional group. The mean body mass index was $24.9 \mathrm{~kg} / \mathrm{m}^{2}$ in the navigation group and $25.4 \mathrm{~kg} / \mathrm{m}^{2}$ in the conventional group (Table I).

Measurement in navigation. Under navigation guidance, the mechanical axis was measured prior to the osteotomy. This and percentage of mechanical axis ${ }^{21}$ were measured following fixation. Measurement data obtained from navigation guidance were compared with radiological measurement data.

Radiological evaluation. Radiological measurements were calculated from full-length weight-bearing anteroposterior radiographs of the leg, including the hip, knee and ankle (an orthoroentgenogram), and a lateral view of the knee using the picture-archiving communication system. The radiological results were evaluated pre- and postoperatively, with respect to the mechanical axis, mechanical axis percentage, femorotibial angle and posterior slope angle of the tibia. The mechanical axis of the femur was defined as the line connecting the centre of the hip and the highest point of the femoral trochlea. The mechanical axis of the tibia was defined as the line connecting the centres of the tibial intercondylar eminences and the talus. The mechanical axis was defined as the angle between femoral and tibial mechanical axes. The mechanical axis percentage was defined as the percentage in which the mechanical axis (Misculicz line) bisects the total width of the tibia (Fig. 1). ${ }^{21}$ The femorotibial angle was defined as the angle between femoral and tibial anatomical axes. ${ }^{18}$ Posterior slope angle was defined as the angle formed by the perpendicular line of the tibial intramedullary reference line and the medial tibial plateau. ${ }^{22}$ The correction angle was defined as the difference between the pre- and the post-operative mechanical axis. The change of posterior slope angle refers to the difference between pre- and post-operative posterior slope 

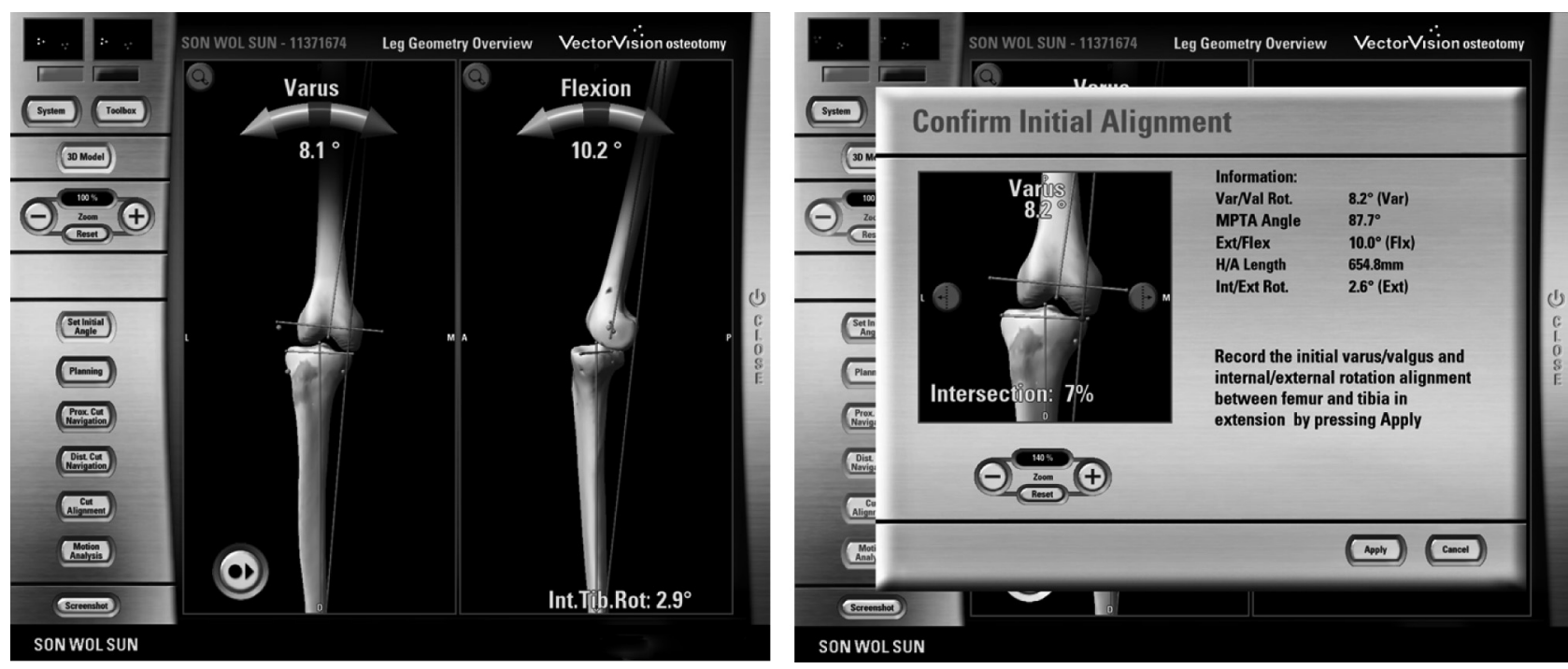

Fig. 2

Screenshots showing the planning overview after registration, the navigation system gives information about the amount of varus deformity.

angle. Two independent investigators (SJS and KHY) performed all radiological measurements to reduce any observation bias. The intra- and interobserver reliability of all the measurements was assessed using the intra-class correlation coefficient. In this study, intra-class correlation coefficient values of all measurements was $>0.861$ for both intra- and interobserver reliability.

Statistical analysis. Reliability was assessed by comparing the measurement data obtained under navigation guidance with the radiological measurements (using Pearson's correlation analysis). The accuracy of the correction was assessed by comparing post-operative mechanical axis, mechanical axis percentage and femorotibial angle of the navigation group with the conventional groups using Student's $t$-test. We also used the same method to compare the change in posterior slope angle between the navigation group and the conventional group following surgery. Variability was assessed by comparing the SD and distribution of mechanical axis between the two groups. Statistical analysis was performed using SPSS version 12.0 (SPSS Inc., Chicago, Illinois) and a p-value $<0.05$ was considered to be significant.

Surgical technique. In the navigation group a transverse incision was made from the tibial tubercle to the bare area of the fibular neck. A dome-shaped fascial incision was then made from the lateral border of the patellar ligament to the edge of Gerdy's tubercle, and extending to the bare area of the fibula. The tibialis anterior muscle was dissected subperiosteally from the tibia and reflected distally. The joint capsule and articular surface between the fibular head and proximal tibia were removed with an osteotome and a pituitary rongeur. Two separate reference bases were then fixed to the distal femur and to the middle tibia with a pair of half-pins. Following this, eight kinematic and anatomi- cal landmarks were registered. These included the kinematic hip centre, the medial and lateral malleoli, the medial and lateral tibial plateau, the medial and lateral femoral epicondyles and the anteroposterior tibial rotation. We judged the start and endpoints to be $15 \mathrm{~mm}$ distal to the tibial plateaux. The software automatically calculates the length of the osteotomy to leave a $3 \mathrm{~mm}$ to $5 \mathrm{~mm}$ medial cortical hinge. The far cortical hinge is best tailored to be $3 \mathrm{~mm}$ in order to help the surgeon to close the wedge. The software sets the cut plane of the osteotomy perpendicular to the mechanical axis according to the sagittal plane. Additional slope can be fine-tuned in the planned osteotomy, in keeping with the posterior slope angle shown in the preoperative radiograph. For the optimum correction the postoperative mechanical axis percentage, which will take place via the navigation system, is $62 \%$. The navigation system provides information about the deformity, level of osteotomy, correction angle and wedge size (Fig. 2). We confirmed these data. A pre-calibrated navigated drill guide was used to place two Kirschner $(\mathrm{K})$-wires in the proximal plane of the osteotomy. Another two K-wires were inserted in the distal plane of the osteotomy in the same manner (Fig. 3). The proximal and distal osteotomies were carried out using an electric saw over the two K-wires. The wedge was removed and the medial far cortex was then carefully decorticated using the saw or an osteotome. A valgus force was then applied slowly to the extremity until the two osteotomy surfaces were firmly opposed to each other. Then, the site was rigidly fixed using a miniplate staple of appropriate size (Fig. 3). ${ }^{4,5}$ The final alignment was confirmed on the computer screen (Fig. 4) using the image intensifier. The placement of the fixation and length of the screw were confirmed and the wound was closed. Postoperatively, isometric exercises of the extensors and the 

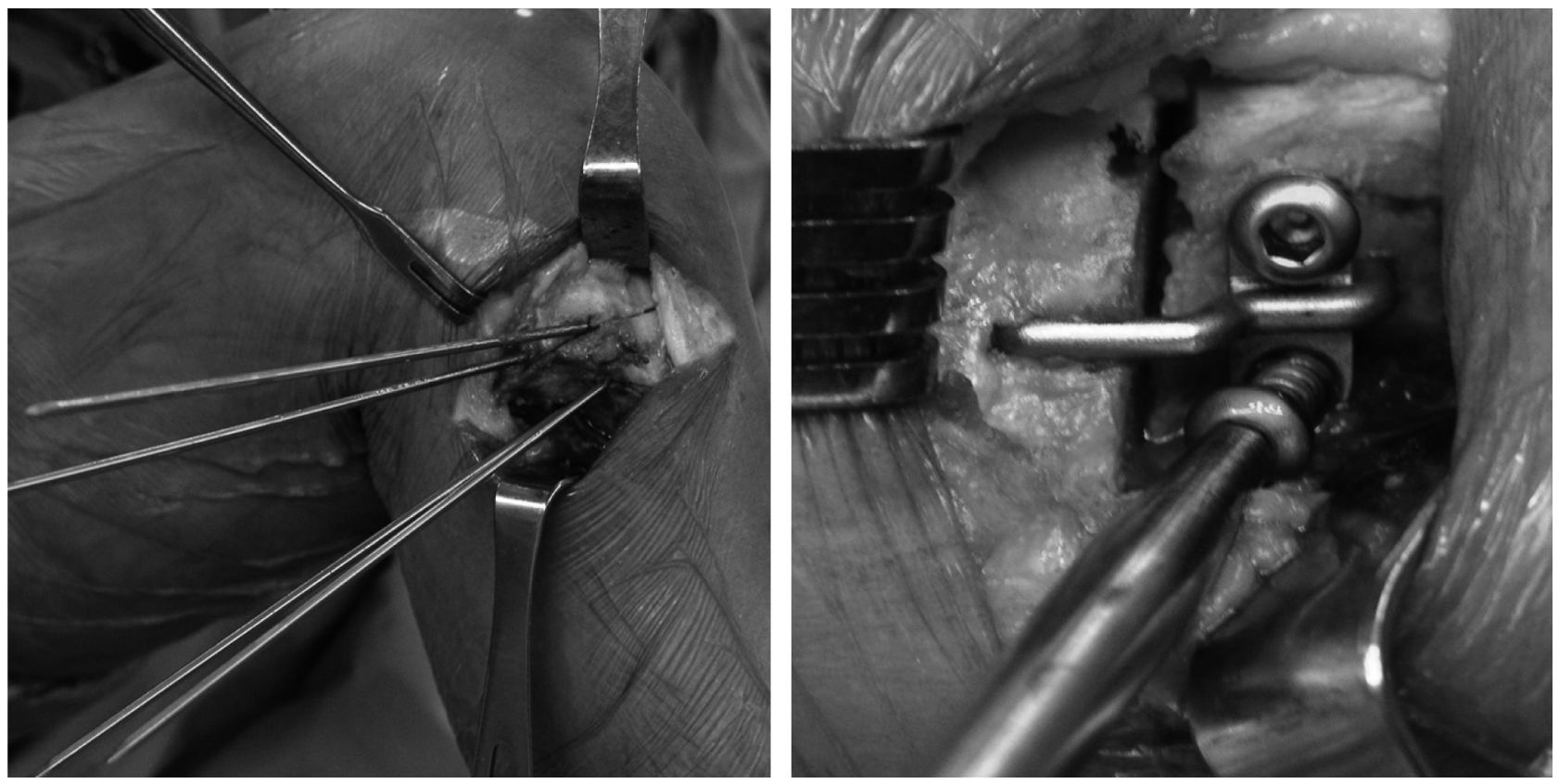

Fig. 3

Intra-operative photographs of the high tibial osteotomy in progress.
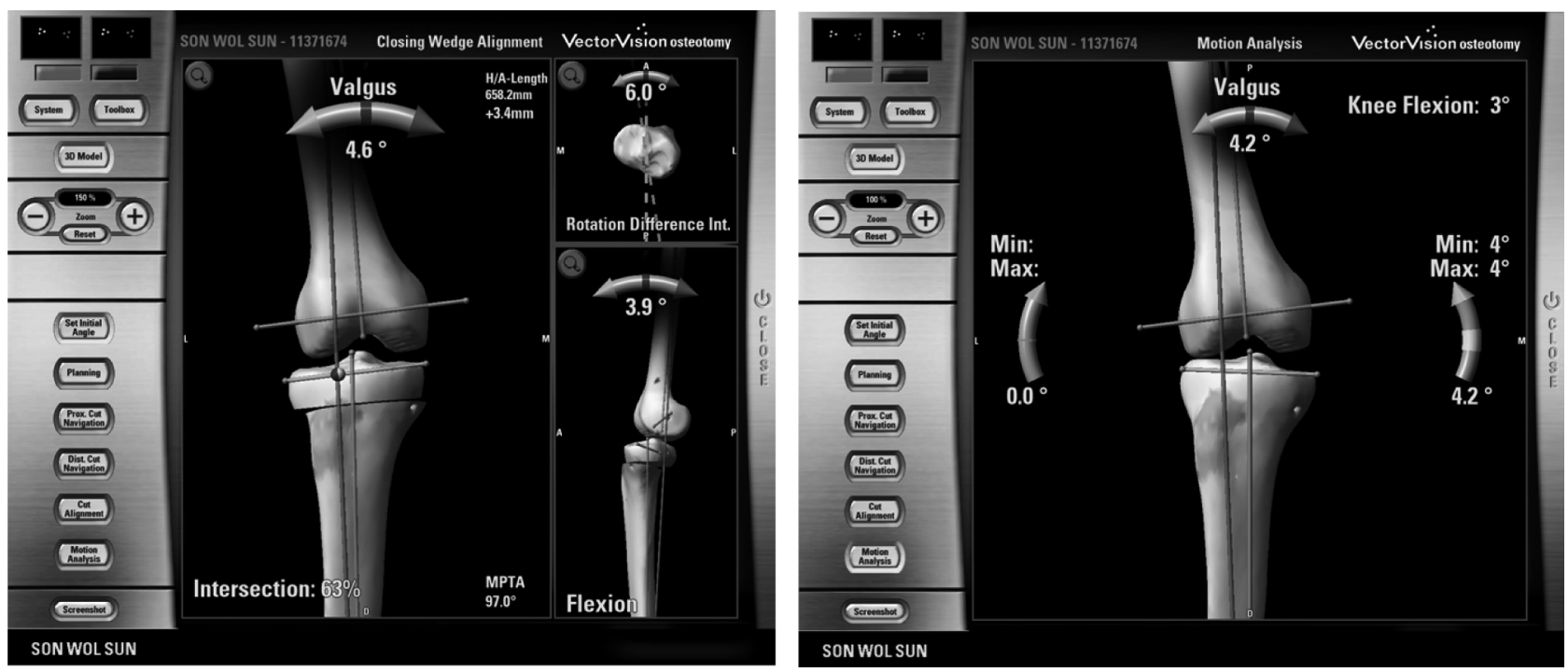

Fig. 4

Screenshots showing how after the closing of the wedge, the limb alignment and confirmed correction angle are assessed in the navigation system.

flexors were commenced. On the second post-operative day, active and passive exercises were commenced. Straight leg raising exercises were initiated on the third postoperative day, and partial weight-bearing at one week. A standing orthoroentgenogram was obtained at two weeks post-operatively and the patient allowed to fully weightbear at six to 12 weeks, depending on their condition.
In the conventional group, the method for determining the correction angle and the wedge size included a preoperative radiological plan, and intra-operative measurement with the help of a cable. ${ }^{23}$ The knee is fully extended and the patella should be facing anteriorly during the intraoperative measurement. With the image intensifier beam strictly vertical, the centre of the femoral head and the 
Table II. Comparison between measured data taken under navigation guidance and by radiographs in the navigated high tibial osteotomy group

\begin{tabular}{lccll}
\hline & Navigation & Radiograph & r-value & p-value \\
\hline Pre-operative $\mathrm{MA}^{*}\left(^{\circ}\right)(\mathrm{SD})$ & $-8.3(2.6)$ & $-7.3(2.6)$ & 0.692 & 0.000 \\
Post-operative $\left.\mathrm{MA}^{\circ}{ }^{\circ}\right)(\mathrm{SD})$ & $3.6(1.3)$ & $2.1(2.3)$ & 0.383 & 0.037 \\
$\mathrm{MA}^{\dagger}(\mathrm{SD})$ & $62.4(5.5)$ & $58.8(10.3)$ & 0.377 & 0.048 \\
\hline
\end{tabular}

* MA, mechanical axis

† MA\%, mechanical axis percentage
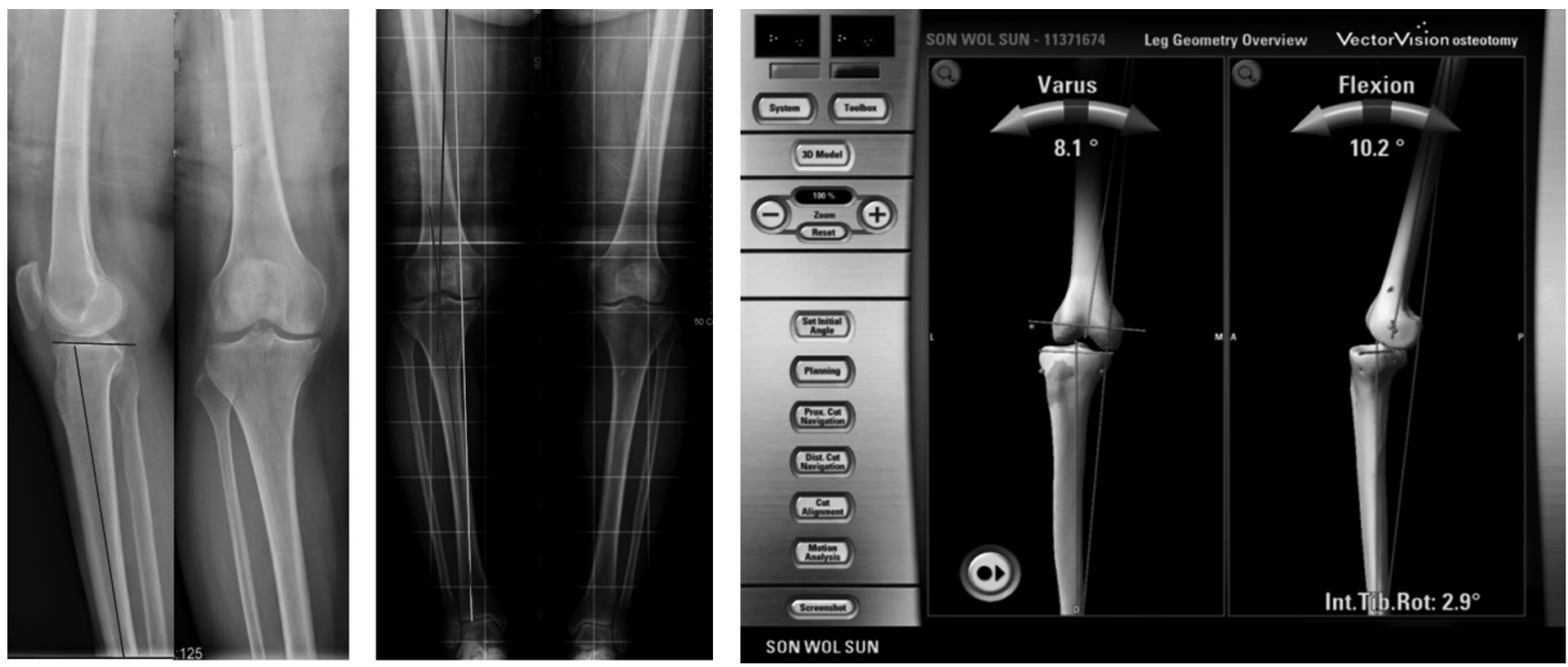

Fig. 5

Comparison between measured data taken under navigation and by radiographs. In the pre-operative roentgenogram of the right knee of a $63-y e a r-$ old woman, the mechanical axis is varus $7.5^{\circ}$ and the mechanical axis percentage is $16.6 \%$. The posterior slope angle of tibia is $8.4^{\circ}$. In the navigation screen, the mechanical axis is varus $8.1^{\circ}$ and the mechanical axis percentage is $7 \%$.

centre of the ankle are marked. A diathermy cable is then placed between these two points and the image intensifier centred on the knee joint. The mechanical axis is determined using the projection of the cable on the image intensifier. For the optimum degree of correction, the postoperative mechanical axis percentage, which will be performed with the image intensifier, is approximately $62 \%$.

\section{Results}

In the navigation group, the mean mechanical axis prior to the osteotomy was varus $8.2^{\circ}$ (SD 2.6) on the computer screen. The mean mechanical axis and mechanical axis percentage after correction were valgus $3.6^{\circ}$ (SD 1.3) and $62.4 \%$ (SD 5.5), respectively. On the radiographs the mean mechanical axis was varus $7.3^{\circ}$ (SD 2.6) pre-operatively. The mean post-operative mechanical axis and mechanical axis percentage were valgus $2.1^{\circ}$ (SD 2.3) and $58.8 \%$ (SD 10.3). There was a positive correlation between the measurement data obtained with the navigation system and that with the radiological measurement $(\mathrm{r}>0.3, \mathrm{p}<0.05)$ (Table II). The mean difference between the post-operative mechanical axis via the navigation system and via the radiograph was $1.5^{\circ}$ (SD 2.3). The mean difference in postoperative mechanical axis percentage between the navigation system and the radiograph was 3.6\% (SD 10.2). The post-operative mechanical axis via the radiological assessment was smaller than via navigation in 37 cases, and greater in 13 cases (Figs 5 and 6).

In the conventional group, the mean mechanical axis was varus $10.6^{\circ}$ (SD 2.9) pre-operatively and valgus $0.1^{\circ}$ (SD 3.7) post-operatively. The mean mechanical axis percentage was $0.7 \%$ (SD 20.4) pre-operatively and $47.3 \%$ (SD 22.1) postoperatively. There were statistically significant differences of post-operative mechanical axis and mechanical axis percentage between the navigation group and the conventional group $(\mathrm{p}=0.001)$ (Table III). The mean pre-operative posterior slope angle was $11.0^{\circ}$ (SD 2.7), which decreased to $9.0^{\circ}$ (SD 3.0) in the navigation group. The mean pre-operative posterior slope angle was $10.4^{\circ}$ (SD 2.8), which decreased to $6.4^{\circ}$ (SD 3.0) in the conventional group $(\mathrm{p}=0.000)$ (Table III). The variability of post-operative mechanical axis was significantly lower in the navigation group $\left(2.3^{\circ} v s 3.7^{\circ}\right.$, $\mathrm{p}=0.012$ ), and the distribution of mechanical axis was also narrower in the navigated group (Table IV). 

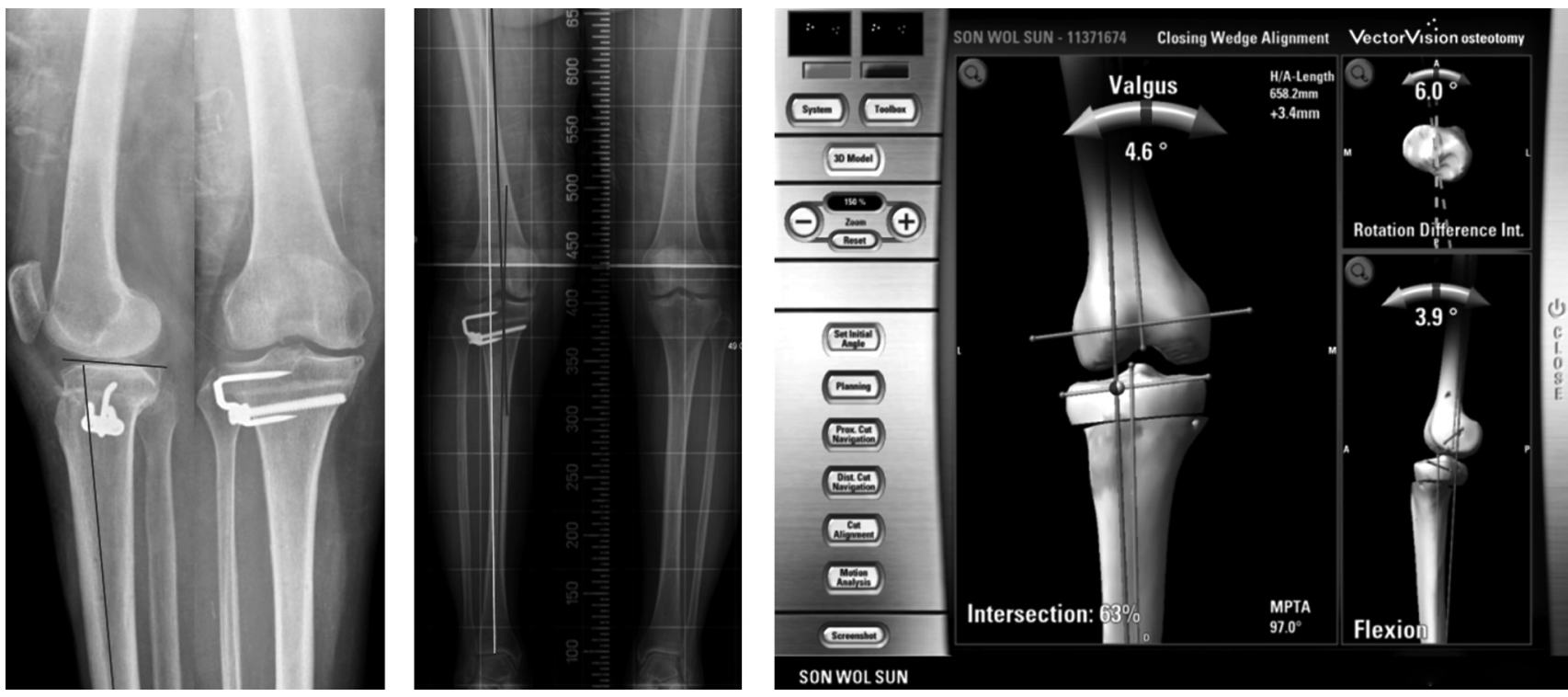

Fig. 6

This patient had a closed wedge high tibial osteotomy using navigation system. On the navigation screen, the mechanical axis is valgus $4.6^{\circ}$ and the mechanical axis percentage is $63.0 \%$. On the 2 nd week post-operative roentgenogram, the mechanical axis is valgus $3.3^{\circ}$ and the mechanical axis percentage is $58.4 \%$. The posterior slope angle is $7.6^{\circ}$. The post-operative mechanical axis and mechanical axis percentage in the roentgenogram are smaller than the post-operative mechanical axis.

Table III. Comparison of radiological measurements between the navigated and the conventional high tibial osteotomy groups

\begin{tabular}{|c|c|c|c|}
\hline & Navigation group & Conventional group & p-value \\
\hline \multicolumn{4}{|l|}{ Pre-operative } \\
\hline $\mathrm{MA}\left({ }^{\circ}\right)(\mathrm{SD})$ & $-7.3(2.6)$ & $-10.6(2.9)$ & 0.000 \\
\hline $\mathrm{MA} \%(\mathrm{SD})$ & $16.9(11.1)$ & $-0.7(20.4)$ & 0.000 \\
\hline FTA $\left(^{\circ}\right)(\mathrm{SD})$ & $-0.6(2.4)$ & $-4.0(4.0)$ & 0.000 \\
\hline PSA $\left({ }^{\circ}\right)(\mathrm{SD})$ & $11.0(2.7)$ & $10.4(2.8)$ & 0.234 \\
\hline \multicolumn{4}{|l|}{ Post-operative } \\
\hline $\mathrm{MA}\left({ }^{\circ}\right)(\mathrm{SD})$ & $2.1(2.3)$ & $0.1(3.7)$ & 0.001 \\
\hline MA\% (SD) & $58.8(10.3)$ & $47.3(22.1)$ & 0.001 \\
\hline FTA $\left(^{\circ}\right)(\mathrm{SD})$ & $8.7(2.6)$ & $6.5(3.7)$ & 0.001 \\
\hline Change of PSA $\left(^{\circ}\right)(\mathrm{SD})$ & $-2.0(2.3)$ & $-4.0(1.2)$ & 0.000 \\
\hline
\end{tabular}

* MA, mechanical axis; MA\%, mechanical axis percentage; FTA, femorotibial angle; PSA, posterior slope angle

\section{Discussion}

The lack of exact intra-operative real-time control of the mechanical axis often results in post-operative malalignment, which is a factor in poor long-term results following a HTO. ${ }^{13,17,24-26}$ This problem can be addressed with the use of a navigation system. The in vitro evaluation of a navigated open-wedge HTO with a plastic bone model suggests that the error of deformity correction is $<1.7^{\circ}(95 \%$ confidence limit (CL) in the frontal and $<2.3^{\circ}(95 \% \mathrm{CL})$ in the sagittal plane, respectively. ${ }^{27}$ The results of a cohort study comparing navigated and conventional HTOs showed a $96 \%$ reproducibility in achieving a mechanical axis of $4^{\circ}$ (SD 2 ) in the navigation group compared to $71 \%$ in the conventional group. ${ }^{28}$ The results of our study comparing navigated with conventional HTOs showed an $86 \%$ reproducibility in achieving a mechanical axis of $3^{\circ}$ (SD 3) in the navigated group, compared to $50 \%$ reproducibility in the conventional group. Therefore, the navigated HTO significantly improved the accuracy and reduced the variability of the correction. Alignment errors are known to occur as a result of limb rotation and knee flexion in conventional HTO because limb alignment is evaluated with a two-dimensional measurement. ${ }^{14,29}$ It has been reported previously that rotation and flexion of $5^{\circ}$ can lead to significant errors in alignment of $3.4^{\circ}$ and $2.8^{\circ}$, respectively. ${ }^{14}$ These discrepancies of the axis due to rotational movements, as well as flexion of the knee joint, can be avoided and corrected immediately with the help of navigation. ${ }^{14} \mathrm{In}$ this study, two independent investigators, performed all the radiological measurements with good intra- and inter- 
Table IV. Distribution of post-operative mechanical axis in the navigated and the conventional high tibial osteotomy groups (number of cases)

\begin{tabular}{lcc}
\hline Post-operative $\mathbf{M A}^{*}\left(^{\circ}\right)$ & Navigated group & Conventional group \\
\hline-6 to -4 & 0 & 5 \\
-4 to -2 & 2 & 14 \\
-2 to 0 & 3 & 4 \\
0 to 2 & 20 & 12 \\
2 to 4 & 18 & 6 \\
4 to 6 & 5 & 7 \\
6 to 8 & 1 & 0 \\
8 to 10 & 1 & 2 \\
Total & 50 & 50 \\
\hline
\end{tabular}

* MA, mechanical axis

observer reliability of all measurements. There were positive correlations between the measurement data for preand post-operative mechanical axis and mechanical axis percentage with the navigation system and with radiological measurement $(\mathrm{r}>0.3, \mathrm{p}<0.05)$.

The mean age of the patients in this study was 60.1 years (45 to 76), which is high by most western standards. However, in Asian countries HTO is considered to be an effective form of surgical treatment that does not limit the oriental lifestyle, such as sitting cross legged and kneeling, and is performed even in older patients with moderate osteoarthritis. Additionally, we considered not only chronological age, but also physiological age and bone quality. The only indication for a TKR in our practice is severe osteoarthritis.

Most patients in this study were women. This gender distribution of osteoarthritis is a common finding in Korean ethnic groups, caused by the racial difference of disease demographics and gender distinction of the incidence of bow leg deformity. ${ }^{6}$

Our post-operative mechanical axis and mechanical axis percentage measurements on the radiographs were less than those under navigation guidance by as much as $1.5^{\circ}$ (SD 2.3) and 3.6\% (SD 10.2). This difference could occur because the post-operative mechanical axis and mechanical axis percentage with the navigation system were measured with patients in a supine position, and the post-operative radiographs were taken in a weight-bearing standing position. An anatomical study using fresh cadaver specimens has been carried out to elucidate this finding. ${ }^{30}$ As the osteotomy site was compressed the lateral collateral ligament became lax in the unloaded state. A varus load increases in the lateral articular joint space, and would tend to exacerbate the loss of correction. ${ }^{30}$ Axial loading of the limb is reported to affect alignment in the navigated limb axis measurements during an HTO. ${ }^{14,20,30}$ Kendoff et $\mathrm{al}^{21}$ reported that the application of a simulated weight-bearing force altered the mechanical axis by up to $2.5^{\circ}$, and so they recommend that this potential change in the axis should be taken into account to avoid under- or overcorrection.

The control of wedge size and accurate placement of the hinge axis are critical aspects of the closed-wedge HTO procedure. Too lateral a placement of the hinge axis can make the closure procedure difficult, or result in a tibial plateau fracture. On the other hand, too medial a placement will weaken the medial cortex, which may result in instability of the fixation and loss of correction. ${ }^{6,18}$ With the conventional technique it is difficult to accurately control the hinge position even with the jig system, because it can only control the wedge size. Three-dimensional orientation of the wedge is important because asymmetrical closure could introduce unwanted secondary deformity, such as malrotation in the transverse plane or alteration of the posterior slope angle in the sagittal plane. ${ }^{14,31}$ An important advantage of the navigation system in closed-wedge HTO is the accurate control of the hinge axis and wedge orientation. ${ }^{14,27}$ The navigated guidance and control of hinge thickness makes the process of wedge closure safe and easy. Therefore, no tibial plateau fractures or fractures of the medial cortex, or failed implant positions were observed in our series either in the conventional or the navigated group. However, the conventional technique required the additional exposure to radiation and meticulous care under fluoroscopic guidance.

One criticism of the computer assisted surgery registration technique is that it may not be accurate in identifying the anatomical landmark percutaneously. We marked the landmark prior to registration with careful attention to surface anatomy.

An asymmetrical closure might produce a significant alteration in the posterior slope angle. This tends to increase after an open-wedge HTO, ${ }^{15,32,33}$ and to decrease after a closedwedge or dome HTO. ${ }^{31,34-38}$ After a closed-wedge HTO the average difference of the posterior slope angle was reported to be $-3.7^{\circ}$ in Lee's study ${ }^{37}$ and $-4.9^{\circ}$ in Hohmann's study. ${ }^{32}$ In our study the mean change of the posterior slope angles were $-2.0^{\circ}(\mathrm{SD} 2.3)$ in the navigation group and $-4.0^{\circ}(\mathrm{SD} 1.2)$ in the conventional group $(p=0.000)$. We believe that accurate orientation of the wedge and proper placement of the hinge axis under navigation guidance could reduce the change in posterior slope angle.

The main limitation of this study is that a prospective cohort of the navigated group was compared with a retrospective matched cohort of the conventional group, rather than setting up a prospective randomised controlled trial.

There were some demographic discrepancies as well, such as the distribution of the operative period, preoperative varus angle, and bilaterality.

The navigated group consisted of 50 procedures performed within a period of 12 months, whereas the retrospective control group consisted of 50 procedures spread over 12 years. The surgeon had experience of using the conventional closed-wedge HTO for more than ten years, and had performed more than 120 cases before 1994 . Therefore, the learning curve of the conventional technique was outside the first cohort period.

We thought that the use of navigation in a closed-wedge HTO might increase the accuracy and precision of the cor- 
rection angle and hence contribute to a better outcome. This led to a renewed interest in closed-wedge HTO since 2006, when the software for using the navigation system was introduced in our hospital. This explains the discrepancy between the two groups in the distribution of the operative period.

In view of the pre-operative varus angle, the mean preoperative mechanical axis percentage was $16.9 \%$ (SD 11.1) in the navigated group and $-0.7 \%$ (SD 20.4) in the conventional group. However, the mean correction angles were $9.4^{\circ}$ (SD 2.7) in the navigated group and $10.8^{\circ}$ (SD 4.6) in the conventional group. Both groups included ideal patients with a correctable deformity considering the selection criteria, and had the same target of post-operative mechanical axis percentage $(62 \%)$.

Two patients in the navigation group had a bilateral closed-wedge HTO, and 16 patients in the conventional group had a bilateral operation. The reason for this difference is that 34 patients in the retrospective control group were spread over 12 years, and 16 patients had a staged operation with a considerable time interval.

We already had sufficient experience of performing a closed-wedge HTO prior to the first cohort period, and we have compared the radiological measurements only at two weeks post-operatively without the clinical outcome. Therefore, the discrepancy in the distribution of the operative period, pre-operative varus deformity and bilaterality should not alter the results.

Knowing that the clinical results of a HTO in varus deformity are related to the extent of the correction of the post-operative valgus alignment, ${ }^{3}$ this study suggests that the use of navigation might contribute to a better clinical outcome. However, future studies are required to identify if the improved accuracy of the navigated surgery provides any clinical advantage.

No benefits in any form have been received or will be received from a commer cial party related directly or indirectly to the subject of this article.

\section{References}

1. Coventry MB, Ilstrup DM, Wallrichs SL. Proximal tibial osteotomy: a critical longterm study of eighty-seven cases. J Bone Joint Surg [Am] 1993;75-A:196-201.

2. Torgerson WR Jr, Kettelkamp DB, Igou RA Jr, Leach RE. Tibial osteotomy for the treatment of degenerative arthritis of the knee. Clin Orthop 1974;101:46-52.

3. Brinkman JM, Lobenhoffer P, Agneskirchner JD, et al. Osteotomies around the knee: patient selection, stability of fixation and bone healing in high tibial osteotomies. J Bone Joint Surg [Br] 2008;90-B:1548-57.

4. Bae DK, Moon KS, Kwon OS. A newly designed miniplate staple for high tibial osteotomy. Bull Hosp Jt Dis 1997;56:167-70.

5. Bae DK, Moon MS, Park BY, Kwon OS. A new design of miniplate staple for high tibual osteotomy: biomechanical study. J Korean Orthop Assoc 1995;30:1433-9.

6. Bae DK, Yoon KH, Kwon OS, Kim YC, Shin DJ. Results and survivorship of high tibial osteotomy. J Korean Orthop Assoc 2002;37:357-63.

7. Bauer GC, Insall J, Koshino T. Tibial osteotomy in gonarthrosis (osteo-arthritis of the knee). J Bone Joint Surg [Am]1969;51-A:1545-63.

8. Akizuki S, Shibakawa A, Takizawa T, Yamazaki I, Horiuchi H. The long-term outcome of high tibial osteotomy: a ten- to 20-year follow-up. J Bone Joint Surg [Br] 2008;90-B:592-6.

9. Hanssen AD. Osteotomy about the knee: american perspective. In: Insall JN, Scott WN, eds. Surgery of the knee. Third ed. New York: Churchill Livingstone, 2001:1447-64.
10. Kirgis A, Albrecht S. Palsy of the deep peroneal nerve after proximal tibial osteotomy: an anatomical study. J Bone Joint Surg [Am] 1992;74-A:1180-5

11. Hsu RW, Himeno S, Coventry MB, Chao EY. Normal axial alignment of the lower extremity and load-bearing distribution at the knee. Clin Orthop 1990;255:215-27.

12. Sprenger TR, Doerzbacher JF. Tibial osteotomy for the treatment of varus gonarthrosis: survival and failure analysis to twenty-two years. J Bone Joint Surg [Am] 2003;85-A:469-74

13. DahI MT. Preoperative planning in deformity correction and limb length surgery. Instr Course Lect 2000;49:503-9.

14. Ellis RE, Tso CY, Rudan JF, Harrison MM. A surgical planning and guidance system for high tibial osteotomy. Comput Aided Surg 1999;4:264-74.

15. El-Azab H, Halawa A, Anetzberger H, Imhoff AB, Hinterwimmer S. The effect of closed- and open-wedge high tibial osteotomy on tibial slope: a retrospective radiological review of 120 cases. J Bone Joint Surg [Br]2008;90-B:1193-7.

16. Kendoff D, Clark M, Pearle A, et al. Influence of lower limb rotation in navigated alignment analysis: implications for high tibial osteotomies. Knee Surg Sports Traumatol Arthrosc 2007;15:1003-8

17. Moreland JR, Bassett LW, Hanker GJ. Radiographic analysis of the axial alignment of the lower extremity. J Bone Joint Surg [Am] 1987;69-A:745-9.

18. Noyes FR, Barber-Westin SD, Hewett TE. High tibial osteotomy and ligament reconstruction for varus angulated anterior cruciate ligament-deficient knees. Am J Sports Med 2000;28:282-96.

19. Wright JG, Treble N, Feinstein AR. Measurement of lower limb alignment using long radiographs. J Bone Joint Surg [Br] 1991;73-B:721-3.

20. Hankemeier S, Hufner T, Wang G, et al. Navigated open-wedge high tibial osteotomy: advantages and disadvantages compared to the conventional technique in a cadaver study. Knee Surg Sports Traumatol Arthrosc 2006;14:917-21.

21. Kendoff D, Board TN, Citak M, et al. Navigated lower limb axis measurements: influence of mechanical weight-bearing simulation. J Orthop Res 2008;26:553-61

22. Jackson DW, Warkentine B. Technical aspects of computer-assisted opening wedge high tibial osteotomy. J Knee Surg 2007;20:134-41.

23. Oswald MH, Jakob RP, Schneider E, Hoogewoud HM. Radiological analysis of normal axial alignment of femur and tibia in view of total knee arthroplasty. J Arthroplasty 1993:8:419-26.

24. Krettek C, Miclau T, Grün O, Schandelmaier P, Tscherne H. Intraoperative control of axes, rotation and length in femoral and tibial fractures: technical note. Injury 1998;29:29-39.

25. Marti CB, Gautier E, Wachtl SW, Jakob RP. Accuracy of frontal and sagittal plane correction in open-wedge high tibial osteotomy. Arthroscopy 2004;20:366-72.

26. Noyes FR, Mayfield W, Barber-Westin SD, Albright JC, Heckmann TP. Opening wedge high tibial osteotomy: an operative technique and rehabilitation program to decreae complications and promote early union and function. Am J Sports Med 2006;34:1262-78.

27. Wang G, Zheng G, Keppler P, et al. Implementation, accuracy evaluation, and preliminary clinical trial of a CT-free navigation system for high tibial opening wedge osteotomy. Comput Aided Surg 2005;10:73-85

28. Keppler P, Gebhard F, Grutzner PA, et al. Computer aided high tibial open wedge osteotomy. Injury 2004;35(Suppl I): 68-78.

29. Saragaglia D, Roberts J. Navigated osteotomies around the knee in 170 patients with osteoarthritis secondary to genu varum. Orthopedics 2005;28(Suppl 10):1269-74.

30. Kawakami H, Sugano N, Yonenobu K, et al. Effects of rotation on measurement of lower limb alignment for knee osteotomy. J Orthop Res 2004;22:1248-53.

31. Shaw JA, Dungy DS, Arsht SS. Recurrent varus angulation after high tibial osteotomy: an anatomic analysis. Clin Orthop 2004;420:205-12.

32. Hohmann E. Bryant A, Imhoff AB. The effect of closed wedge high tibial osteotomy on tibial slope: a radiographic study. Knee Surg Sports Traumatol Arthrosc 2006;14:454-9.

33. Nakamura E, Mizuta H, Kudo S, Takagi K, Sakamoto K. Open-wedge osteotomy of the proximal tibia hemicallotasis. J Bone Joint Surg [Br] 2001;83-B:1111-15.

34. Naudi DD, Amendola A, Fowler PJ. Opening wedge high tibial osteotomy for symptomatic hyperextension-varus thrust. Am J Sports Med 2004;32:60-70.

35. Giffin JR, Vogrin TM, Zantop T, Woo SL, Harner CD. Effects of increasing tibial slope on the biomechanics of the knee. Am J Sports Med 2004;32:376-82.

36. Kaper BP, Bourne RB, Rorabeck CH, Macdonald SJ. Patella infera after high tibial osteotomy. J Arthroplasty 2001;16:168-73.

37. Lee JY, Seon JK, Song EK, et al. Comparison of high tibial osteotomy: opening versus closing wedge osteotomy. J Korean Orthop Assoc 2004;39:790-6.

38. Lerat JL, Moyen B, Garin C, et al. Anterior laxity and internal arthritis of the knee: results of the reconstruction of the anterior cruciate ligament associated with tibial osteotomy. Rev Chir Orthop Reparatrice Appar Mot 1993;79:365-74 (in French). 